\title{
Tratamiento quirúrgico de la endometriosis pélvica profunda con compromiso colorrectal
}

\author{
Eduardo Mordojovich Z. ${ }^{1}$, Leonardo Espíndola S. ${ }^{1}$, Ernesto Melkonian T. ${ }^{1}$, \\ Daniella Espínola M. ${ }^{1}$, Constanza Villalón M. ${ }^{1}$, Christian Jensen B. ${ }^{1}$, \\ Aldo Cuneo Z. ${ }^{1}$, Marco Puga A. ${ }^{2}$, César Sandoval S. ${ }^{2}$ e Ignacio Miranda M. ${ }^{2}$
}

\section{Surgical treatment of deep pelvic endometriosis with colorectal involvement}

Introduction: In endometriosis, intestinal involvement affects up to $12 \%$ of patients, compromising the rectum and the rectosigmoid junction in $90 \%$ of cases. Aim: Describe the experience of the Coloproctolgy and Gynecology Team of the Clínica Alemana de Santiago in the surgical treatment of deep pelvic endometriosis with colorectal involvement. Materials and Methods: Retrospective study based on the database of patients with endometriosis treated between January 2015 and April 2018. The inclusion criteria were patients with deep pelvic endometriosis clinic, who had colorectal involvement and who had been treated with rectal shaving, discoid resection or segmental resection. Electronic clinical records, operative protocols and definitive biopsies were reviewed. Results: Twenty-five patients with a median age of 35 years were recruited. The main symptom of consultation was dysmenorrhoea and the most frequent digestive symptom was dyschezia. In 8 patients a rectal shaving was performed, in 7 a sigmoidectomy, in 9 a discoid resection and in 1 patient a tiflectomy. The only reported post-operative complication was low gastrointestinal bleeding in 4 of the 25 patients (Clavien-Dindo I and IIIa). A median follow-up of 13 months was achieved, to date 3 patients have been diagnosed with some type of recurrence. Conclusion: It is important that the surgery to be performed guarantees low morbidity and recurrence. The results in our center are encouraging, which makes us believe that surgical treatment could be a good alternative in deep pelvic endometriosis with colorectal involvement.

Key words: colorectal endometriosis; deep endometriosis; shaving; disc excision.

\section{Resumen}

Introducción: En la endometriosis el compromiso intestinal afecta hasta al 12\% de las pacientes, comprometiendo al recto y a la unión rectosigmoidea en el 90\% de los casos. Objetivo: Describir la experiencia del equipo de Coloproctología y Ginecología de Clínica Alemana de Santiago en el tratamiento quirúrgico de la endometriosis pélvica profunda con compromiso colorrectal. Material y Métodos: Estudio retrospectivo a partir de la base de datos de pacientes con endometriosis tratados entre enero del año $2015 \mathrm{y}$ abril de 2018. Los criterios de inclusión fueron pacientes con clínica de endometriosis pélvica profunda, que tuviesen compromiso colorrectal y hayan sido tratados con shaving rectal, resección discoide o resección segmentaria. Se revisaron fichas clínicas electrónicas, protocolos operatorios y biopsias definitivas. Resultados: Se reclutaron 25 pacientes con una mediana de edad de 35 años. El síntoma principal de consulta fue dismenorrea y el síntoma digestivo más frecuente fue disquecia. En 8 pacientes se realizó un shaving rectal, en 7 una sigmoidectomía, en 9 una resección discoide y en 1 paciente una tiflectomía. La complicación posoperatoria reportada fue la hemorragia digestiva baja en 4 pacientes (Clavien-Dindo I y IIIa). Con una mediana de seguimiento de 13 meses, a la fecha 3 pacientes se les ha diagnosticado algún tipo de recidiva. Conclusión: Es importante que la cirugía a realizar, garantice una morbilidad y recurrencia baja. Los resultados en nuestro centro son alentadores, lo que nos hace creer que el tratamiento quirúrgico podría ser una buena alternativa en la endometriosis pélvica profunda con compromiso colorrectal.

Palabras clave: endometriosis colorrectal; endometriosis profunda; shaving; resección discoide.
'Equipo de Coloproctología Clínica Alemana. Santiago, Chile.

Equipo de Ginecología Clínica Alemana. Santiago, Chile.

Recibido el 27 de junio de 2018 y aceptado para publicación el 7 de agosto de 2018.

Correspondencia a: Dr. Eduardo Mordojovich Z. edumordo@gmail.com 


\section{Introducción}

La endometriosis es una patología que se caracteriza por la presencia de glándulas y estroma endometrial fuera del útero. Afecta aproximadamente al $10 \%$ de las mujeres en edad fértil ${ }^{1}$, alcanzando hasta un $50 \%$ en mujeres que consultan por dolor y/o infertilidad ${ }^{2}$. La endometriosis profunda se define arbitrariamente cuando existe compromiso peritoneal mayor a $5 \mathrm{~mm}^{1}$. El compromiso intestinal es una presentación severa de la enfermedad y afecta del 5,3 al $12 \%$ de las pacientes con endometriosis $\mathrm{y}$, generalmente, compromete al recto y a la unión rectosigmoidea en el $90 \%$ de los casos $^{3}$.

La sintomatología clásica es dolor abdominal y pélvico progresivo asociado o no a infertilidad, que llega a afectar la calidad de vida de las pacientes. El tratamiento médico es inefectivo, con alta recurrencia $(76 \%)$ y el tratamiento quirúrgico del compromiso intestinal es controversial, por la potencial morbilidad de la resección colorrectal ${ }^{4}$. Diferentes tipos de cirugía se han descrito para la endometriosis rectosigmoidea, pudiéndose clasificar en resección parcial de la pared rectal (shaving), resección de espesor total de la pared (resección discoide) y resección segmentaria (sigmoidectomía) ${ }^{4}$. Estudios recientes han avalado la seguridad y factibilidad de la resección colorrectal laparoscópica en endometriosis pélvica profunda ${ }^{4}$. A pesar de esto, aún existe poca evidencia nacional publicada con respecto a este tema ${ }^{5-7}$

El objetivo principal de este trabajo es describir la experiencia del equipo de Coloproctología y Ginecología de Clínica Alemana de Santiago en el tratamiento quirúrgico de la endometriosis pélvica profunda con compromiso colorrectal evaluando el tipo de cirugía realizada, las complicaciones reportadas, la remisión sintomática y la presencia o no de recidiva durante el seguimiento.

\section{Materiales y Método}

Estudio retrospectivo a partir de la base de datos de pacientes con endometriosis tratados por el equipo de Ginecología y Coloproctología de Clínica Alemana de Santiago entre enero del año 2015 y abril del año 2018. Los criterios de inclusión fueron: pacientes con clínica de endometriosis pélvica profunda, confirmada por imágenes, estudio endoscópico o de forma intraoperatoria, que tuviesen compromiso de colon y/o recto y que hayan sido tratados de forma quirúrgica. La elección de la técnica a realizar fue caso a caso definida conjuntamente entre el equipo de Ginecología y Coloproctología según las imágenes y los hallazgos intraoperatorios, de acuerdo a los siguientes criterios: en lesiones superficiales con compromiso exclusivo de la serosa se realizó shaving, en lesiones de pared completa con una extensión menor a $3 \mathrm{~cm}$ se realizó una resección discoide y en lesiones de pared completa con una extensión mayor a $3 \mathrm{~cm}$ se realizó una resección segmentaria.

Se revisaron las fichas clínicas electrónicas obteniendo datos clínicos, sintomatología ginecológica y digestiva, búsqueda de embarazo, protocolos quirúrgicos, presencia de complicaciones posoperatorias evaluadas según la clasificación de Clavien-Dindo ${ }^{8}$, días de hospitalización, informes de las biopsias diferidas y el seguimiento clínico de las pacientes.

\section{Resultados}

Se reclutaron 25 pacientes que cumplían los criterios de inclusión. La mediana de edad fue de 35 años con un rango de 25 a 50 años. El síntoma principal de consulta fue la dismenorrea en 22 pacientes, seguido de dispareunia en 2 pacientes y rectorragia en 1 paciente. Nueve de las 25 pacientes consultaban, además, por infertilidad. De los síntomas digestivos, el más prevalente fue la disquecia en 10 pacientes seguido por diarrea catamenial en 6 , rectorragia en 3 y constipación en 3 pacientes. Sólo 3 de las 25 pacientes no presentaba síntomas digestivos (Tabla 1).

En relación al estudio preoperatorio mediante imágenes, 21 de las 25 pacientes se estudió con una resonancia magnética (RM), 3 se estudiaron con ecografía transvaginal y 1 paciente con tomografía computada. En 22 pacientes el compromiso colorrectal se sospechó preoperatoriamente, en los otros 3 casos las imágenes no demostraron compromiso rectal (2 casos de RM y otro de ecografía),

Tabla 1. Síntomas de consulta

\begin{tabular}{|lc|}
\hline Síntomas & Frecuencia \\
\hline Principal & \\
Dismenorrea & $88 \%$ \\
Dispareunia & $8 \%$ \\
Rectorragia & $4 \%$ \\
Digestivo & \\
Disquecia & $40 \%$ \\
Diarrea catamenial & $24 \%$ \\
Rectorragia & $12 \%$ \\
Constipación & $12 \%$ \\
\hline
\end{tabular}


ARTÍCULO ORIGINAL

Tabla 2. Tipo de cirugía y complicaciones reportadas

\begin{tabular}{|c|c|c|c|c|}
\hline Cirugía & $\mathbf{n}$ & Mediana días hospitalización & n de complicaciones & Tipo de complicación \\
\hline Sigmoidectomía lap & 7 & 5 & 1 & Sangrado (Clavien-Dindo IIIa) \\
\hline Shaving rectal & 8 & 3 & 0 & - \\
\hline Resección discoide & 7 & 3 & 2 & Sangrado (Clavien-Dindo I) \\
\hline Doble discoide & 2 & 5 & 1 & Sangrado (Clavien-Dindo I) \\
\hline
\end{tabular}

Tabla 3. Recidiva de endometriosis durante seguimiento

\begin{tabular}{|ccccc|}
\hline Paciente $\mathbf{N}^{\mathbf{0}}$ & Cirugía & Meses seguimiento & Estado actual & Tipo de recidiva \\
\hline 1 & Sigmoidectomía lap & 34 & Asintomática & Quiste endometriósico ovario \\
2 & Shaving rectal & 22 & Asintomática & Quiste endometriósico ovario \\
3 & Resección Discoide & 21 & Dismenorrea & Nódulo rectosigmoideo \\
\hline
\end{tabular}

confirmándose el compromiso intestinal posteriormente como hallazgo intraoperatorio. A 17 de las 25 pacientes se les realizó colonoscopia previo a la cirugía, siendo normal en 2 de estas 17 pacientes. La gran mayoría de las colonoscopias fueron informadas con engrosamiento de la pared rectal o con compresión extrínseca rectal. A las 8 pacientes restantes no se les realizó estudio endoscópico previo a la cirugía.

De las cirugías realizadas, en 8 pacientes se realizó un shaving rectal, en 7 casos una sigmoidectomía, en 9 casos una resección discoide y en 1 paciente una tiflectomía por compromiso del ciego. Todas las cirugías fueron realizadas mediante el abordaje laparoscópico. No se registraron complicaciones intraoperatorias. La complicación posoperatoria reportada fue la hemorragia digestiva baja en 4 de los 25 pacientes (16\%). De las 4 pacientes que presentaron sangrado, 3 fueron tras una resección discoide y 1 luego de una sigmoidectomía. Sólo el caso de la sigmoidectomía requirió de una mayor intervención, realizándose una colonoscopía y controlando el sangrado mediante clips, clasificando esta complicación como IIIa según Clavien-Dindo ${ }^{8}$. El resto de los pacientes con sangrado se manejó de forma conservadora exitosamente (Clavien-Dindo grado I) (Tabla 2).

La mediana de días de hospitalización fue de 4 días, siendo la menor la del shaving rectal con 3 días, y la mayor la sigmoidectomía con 5 días de hospitalización (Tabla 2).

Todas las biopsias diferidas confirmaron focos de endometriosis en la pieza operatoria.
Se logró realizar un seguimiento al $100 \%$ de los pacientes, con una mediana de seguimiento de 13 meses (0 a 34 meses). En la actualidad 21 de las 25 pacientes se encuentran asintomáticas y sólo 4 presentan algún tipo de síntoma (3 pacientes dismenorrea y 1 con dispareunia). A 3 pacientes se les ha diagnosticado recidiva de la endometriosis pelviana, dos de ellas asintomáticas, pero con la presencia de quiste ovárico endometriósico en la ecografía de control y otra paciente con dismenorrea y presencia de recidiva con compromiso rectosigmoideo en la resonancia magnética de control (Tabla 3). A la fecha, 3 de las 9 pacientes que consultaban por infertilidad se han embarazo.

\section{Discusión}

La endometriosis pélvica profunda es un tipo de endometriosis que se presenta, en la mayoría de los casos, como un cuadro clínico de dolor pelviano de gran intensidad asociado a síntomas gastrointestinales bajos y altas tasas de infertilidad, afectando en muchos casos la calidad de vida de los pacientes 9 .

El diagnóstico por definición es histológico, sin embargo, para un adecuado enfrentamiento de la enfermedad se debe recabar información en 3 etapas distintas: historia clínica, examen físico y estudio de imágenes ${ }^{10}$. Debido al carácter de multifocalidad de la endometriosis es importante conocer exactamente la localización de las lesiones colorrectales para así planificar la mejor cirugía posible. Se recomiendan fuertemente en la literatura la endosonografía trans- 
rrectal y la resonancia magnética para estudio de la endometriosis, sin recomendar una sobre la otra. En nuestra serie la gran mayoría de nuestras pacientes (21 de 25) se estudiaron con resonancia magnética de pelvis y ninguna con endosonografía transrectal. Principalmente, se prefirió este método de estudio ya que permite reconocer lesiones colorrectales altas, evaluar al mismo tiempo el compartimento anterior $\mathrm{y}$ posterior ${ }^{10}$ y por no ser operador dependiente. Otro examen complementario usualmente solicitado en este tipo de pacientes es la colonoscopía. Este examen nos permite identificar lesiones transmurales o lesiones extensas con compresión extrínseca. Además, permite realizar el diagnóstico diferencial con tumores malignos en pacientes con hemorragia digestiva baja $^{7}$. En nuestro grupo, a 8 pacientes no se les realizó estudio endoscópico ya que solo se sospechaba compromiso superficial de la pared colorrectal de acuerdo al estudio imagenológico (6 de estas 8 pacientes se les realizó solo shaving). Actualmente, la conducta del equipo es solicitar colonoscopía a todas las pacientes con endometriosis pélvica profunda, independiente de la profundidad aparente del compromiso colorrectal.

Debido al poco éxito del tratamiento médico es que la alternativa quirúrgica ha cobrado fuerza. A pesar de esto, la indicación quirúrgica es aún controversial, debido principalmente a la morbilidad asociada a una resección colorrectal. En la actualidad, muchos equipos de cirujanos realizan resecciones colorrectales en endometriosis profunda ya que creen que con esto disminuirían la recurrencia de la enfermedad ${ }^{11}$. Por otra parte una cirugía conservadora, en que se preserve el recto, llevaría a un mejor resultado funcional desde el punto de vista digestivo. Es por esto que se ha dividido la cirugía de la endometriosis con compromiso colorrectal en resección radical y en resección conservadora, incluyendo a esta última al shaving rectal y a la resección discoide ${ }^{12}$. En nuestro grupo de pacientes se incluyen estos 3 tipos de cirugía. La decisión de qué cirugía realizar fue tomada según los hallazgos a las imágenes $\mathrm{y}$, particularmente, los hallazgos en el intraoperatorio, según los criterios antes señalados. A pesar de esto, aún no existe un consenso acerca de qué cirugía realizar en cada caso ${ }^{4}$. Esta decisión fue tomada de manera multidisciplinaria entre ginecólogos y coloproctólogos, previa conversación con la paciente acerca de los eventuales riesgos de la resección colorrectal (riesgo de filtración de anastomosis y de ostomía).

De los 7 casos de sigmoidectomía, 6 presentaron gran compromiso en el estudio imagenológico, con nódulos mayores a $3 \mathrm{~cm}$ y, además, deformación y estenosis colónica al estudio endoscópico. Solo una paciente a la que se le realizó sigmoidectomía presentó una colonoscopía normal. En pacientes con nódulos de menor tamaño $(<3 \mathrm{~cm})$ pero con compromiso de toda la pared colorrectal se logró realizar exitosamente la resección discoide. En dos casos, con nódulos de $3 \mathrm{~cm}$ se realizó sin incidentes una doble resección discoide. En el resto de los casos, cuando no se observaba gran compromiso de la pared intestinal se logró sin complicaciones la realización del shaving rectal, comprobando posteriormente mediante prueba neumática la hermeticidad de la pared rectal.

En cuanto a las complicaciones post operatorias, en nuestra serie están dadas por el sangrado post operatorio en 4 de las 25 pacientes (16\%). El sangrado digestivo se trató de forma conservadora en 3 casos y mediante un procedimiento mínimamente invasivo en 1 caso.

En la literatura encontramos artículos que evalúan la morbilidad de la resección discoide. Roman y cols. ${ }^{11}$, publican una tasa de complicaciones en resección discoide de $26 \%$, con 2 casos de fístula rectovaginal. Fanfani y cols. ${ }^{4}$, presentan 48 pacientes con resección discoide, con una tasa de complicaciones severas de $12,5 \%$, entre ellas sangrado masivo que requiere transfusiones, 1 paciente con una fístula rectovaginal y 2 casos de reoperaciones. Bray-Beraldo ${ }^{13}$, describe sus resultados en 32 pacientes que se sometieron a resección colorrectal por endometriosis (shaving rectal, resección segmentaria de recto, resección discoide de recto, rectosigmoidectomía, apendicetomía y shaving de ileon). Reporta sólo 2 complicaciones, una fístula anastomótica colorrectal y una fístula recto vaginal que requiere reoperación e ileostomía. En nuestra serie presentamos un $16 \%$ de complicaciones, sin embargo, no tuvimos ningún caso de fístula rectovaginal ni filtración anastomótica ni tampoco ningún caso de reoperación. Ninguna de nuestras 25 pacientes requirió de ostomía.

En cuanto a la mejoría sintomática, Stepniewska ${ }^{9}$, reporta una mejoría completa de la dismenorrea en el $76 \%$ de las pacientes que fueron a resección colorrectal. En nuestra serie, observamos que 21 de las 25 pacientes $(84 \%)$ se presentan asintomáticas luego de la cirugía, con una mediana de seguimiento de 13 meses. Parece interesante evaluar la recurrencia de la sintomatología a largo plazo en nuestra serie, que se describe en la literatura de un $13 \%{ }^{9}$.

Una limitación de nuestro trabajo es no haber realizado estudio endoscópico a todas las pacientes. A las pacientes reclutadas inicialmente y con sospecha de endometriosis con compromiso superficial de 
la pared colorrectal no se les realizó colonoscopía, no pudiendo evaluar el compromiso transmural de la endometriosis ni tampoco descartar diagnósticos diferenciales. Como se describió anteriormente, la conducta actual es realizar estudio endoscópico a todas las pacientes con endometriosis pélvica profunda y sospecha de compromiso colorrectal. Otra limitación de nuestro trabajo es el escaso tiempo de seguimiento (solo 13 meses). Con un mayor tiempo de seguimiento podríamos sacar mejores conclusiones respecto a la remisión sintomática y a la eventual recidiva de la endometriosis en nuestro grupo de pacientes.

\section{Conclusión}

Para un tratamiento exitoso de la endometriosis profunda, es de extrema importancia que el enfoque sea multidisciplinario. Una evaluación ginecológica y coloproctológica adecuada permitirá definir el estudio necesario y la cirugía de elección. Es importante que la cirugía a realizar, ya sea con o sin resección colorrectal, garantice una morbilidad aceptable y una baja recurrencia de la enfermedad. Los resultados en nuestro centro son alentadores, con una baja morbilidad y una alta tasa de regresión sintomática, lo que nos hace creer que el tratamiento quirúrgico podría ser una buena alternativa en la endometriosis pélvica profunda con compromiso colorrectal.

\section{Responsabilidades éticas}

Protección de personas y animales. Los autores declaran que para esta investigación no se han realizado experimentos en seres humanos ni en animales.

Confidencialidad de los datos. Los autores declaran que en este artículo no aparecen datos de pacientes.

Conflictos de interés: no hay.

\section{Referencias}

1. Sourial S, Tempest N, Hapangama DK. Theories on the Pathogenesis of Endometriosis. Int J Reprod Med. 2014;2014:179515.

2. Giudice LC, Kao LC. Endometriosis. Lancet. 2004;364:1789-99

3. Darai E, Dubernard G, Coutant C, Frey C, Rouzier R, Ballester M. Randomized trial of laparoscopic assisted versus open colorectal resection for endometriosis: morbidity, symptoms, quality of life, and fertility. Ann Surg. 2010;251:1018-23.

4. Fanfani F, Fagotti A, Gagliardi ML, Ruffo G, Ceccaroni M, Cambia G, et al. Discoid or segmental rectosigmoid resection for deep infiltrating endometriosis: a case-control study. Fertil Steril. 2010;94:444-9.

5. Prado J, Larraín D, González F, Marengo F, Prado T, Rabischong B, et al. Técnica laparoscópica reversa en el manejo quirúrgico de la endometriosis profunda del tabique rectovaginal: experiencia preliminar. Rev Chil Obstet Ginecol. 2012;77:29-34.

6. Durruty G, Celle C, Zamboni M, Pomés C. La cirugía laparoscópica por endometriosis profunda es efectiva en el manejo del dolor. Rev Chil Obstet Ginecol. 2016;81:189-93.

7. Durruty G, Larraín D, Cuello M, Pomés C, Vidal H, Vergara, A, et al. Endometriosis profunda del tabique rectovaginal con compromiso intestinal: manejo quirúrgico con resección segmentaria de rectosigmoides. Rev Chil Obstet Ginecol. 2008;73:192-203.

8. Dindo D, Demartines N, Clavien PA. Classification of surgical complications: a new proposal with evaluation in a cohort of 6336 patients and result of a survey. Ann Surg. 2003;238:93-6.

9. Stepniewska A, Pomini P, Guerriero M, Scioscia M, Ruffo G, Minelli L. Colorectal endometriosis: benefits of long- term follow up in patients who underwent laparoscopic surgery. Fertil Steril. 2010;93:2444-6.

10. Chapron C, Chopin N, Borghese B, Malartic C, Decuypere F, Foulot H. Surgical Management of deeply Infiltrating Endometriosis: An update. Ann N Y Acad Sci. 2004;1034:326-37.

11. Roman H, Abo C, Huet E, Bridoux V, Auber M, Oden S, et al. Full-Thickness Disc Excision in Deep Endometriotic Nodules of the Rectum: A Prospective Cohort. Dis Colon rectum. 2015;58:957-66.

12. Roman H, Vassilieff M, Tuech JJ, Huet E, Savoye G, Marpeau L, et al. Postoperative digestive function after radical versus conservative surgical philosophy for deep endometriosis infiltrating the rectum. Fertil Steril. 2013;99;1695-704.

13. Bray-Beraldo F, Pereira AMG, Gazzo C, Santos MP, Lopes RGC. Surgical treatment of intestinal endometriosis: Outcomes of three different techniques. Rev Bras Ginecol Obstet. 2018;40:390-96. 